\title{
Equilibrium grain boundary segregation and clustering of impurities in colloidal polycrystalline monolayers
}

\author{
François A. Lavergne, ${ }^{*}$ Samuel Diana, Dirk G. A. L. Aarts, and Roel P. A. Dullens \\ Department of Chemistry, Physical and Theoretical Chemistry Laboratory, University of \\ Oxford, South Parks Road, Oxford, OX1 3QZ, United Kingdom. \\ E-mail: francois.lavergne@chem.ox.ac.uk
}

\section{Abstract}

We investigate the segregation of impurities to grain boundaries in colloidal polycrystalline monolayers using video-microscopy. A model colloidal alloy is prepared by embedding large spherical impurities in a polycrystalline monolayer of small host colloidal hard spheres, which stops grain growth at a finite grain size. The size ratio between the impurities and the host particles determines whether they behave as interstitial or substitutional impurities in the bulk crystal, akin to those in real alloys. We find that the partitioning of impurities between the grains and the grain boundaries is in very good agreement with the Langmuir-McLean adsorption model for equilibrium grain boundary segregation. This enables the direct measurement of the free energy of adsorption for the two types of impurities. Near saturation, we characterise the spatial distribution of the adsorbed impurities and find that it strongly depends on their interstitial or substitutional nature. This is because the relative importance of clustering and mixing due to non-additivity is determined by geometrical constraints imposed by the crystalline host lattice.

\section{Introduction}

The process of alloying, i.e. the incorporation of solute atoms of one or several other elements in a metal, can dramatically change the properties of a material. ${ }^{1}$ For example, solute atoms, or impurities, can interact with grain boundaries (GBs) via impurity $\operatorname{drag}^{2,3}$ or Zener pinning, ${ }^{3-5}$ which stops the grain growth process at a finite grain size. ${ }^{1,6,7}$ Controlling the grain size is key in tuning the mechanical properties of a material due to the Hall-Petch effect, which predicts that the strength of a material sensitively depends on the grain size. ${ }^{8-10}$

In a polycrystalline alloy, impurities tend to be adsorbed at GBs, as they offer more free volume than the grains, a phenomenon known as GB segregation. ${ }^{11,12}$ This is analogous to the adsorption of a gas onto a solid surface, with the impurity concentration in the bulk crystal being akin to the pressure of the gas. ${ }^{11,13,14} \mathrm{At}$ equilibrium, in the ideal case where there are no interactions between adsorbed atoms, the surface coverage as a function of the pressure in the gas phase is given by the Langmuir adsorption model. ${ }^{15,16}$ The counterpart of this model for the case of GB segregation is the McLean model, ${ }^{11}$ which considers impurities distributed on a lattice comprising of crystal sites and GB sites. The fractions of crystal and GB sites occupied by impurities are denoted by $X_{b}$ and $X_{g b}$, respectively. At equilibrium, these two quantities are related to each other via the Langmuir-McLean isotherm,

$$
\frac{X_{g b}}{X_{g b}^{s a t}}=\frac{K X_{b}}{1+K X_{b}},
$$


where $X_{g b}^{s a t}$ is the value of $X_{g b}$ at saturation and $K=\exp \left(e_{a} / k_{B} T\right)$ the equilibrium constant, with $e_{a}$ being the free-energy of adsorption. In the limit of small $X_{b}, X_{g b}$ is expected to increase linearly with $X_{b}$ as the denominator in Eq. (1) is close to unity. Good agreement of this model with experimental data was found for different alloys. ${ }^{17,18}$ Nevertheless, different behaviours can be observed depending on e.g. the solute-solvent interactions, the solutesolvent size ratio and the lattice structure. ${ }^{12,14}$

Colloidal polycrystalline systems with embedded impurities have already been proven to be good model systems to directly visualise GB segregation using brightfield or confocal microscopy. ${ }^{19-22}$ The impurities are usually colloidal particles with a different size than that of the host particles. Such systems have been used to investigate grain size control ${ }^{20,23}$ and the dynamics of segregation during crystallisation in great detail. ${ }^{24-26}$ Monolayers of colloidal spheres with added impurities of a different size are also very interesting from a more fundamental point of view as they provide a simple realisation of a non-additive binary system. ${ }^{27,28}$ Such mixtures exhibit very rich phase behaviour caused by the clustering of the impurity particles due to depletion effects. ${ }^{27-30}$

In this work, we study GB segregation in a polycrystalline monolayer of hard spheres with embedded impurities using video-microscopy. We use impurities of two different sizes and show that their size ratio with the host particles determines whether they behave as interstitial or substitutional impurities in the bulk crystal. We find that their segregation behaviour is in very good agreement with the LangmuirMcLean model of equilibrium adsorption and directly measure the free energy of adsorption in both cases. The spatial distribution of impurities close to saturation highlights different relative contributions of clustering and mixing due to non-additivity depending on the type of impurities. These differences are shown to originate from the geometrical constraints imposed by the lattice.

\section{Methods}

\section{Preparation of "colloidal alloys"}

Our experimental system consists of colloidal polycrystalline monolayers with added larger colloidal particles, termed impurities. The host particles forming the polycrystalline monolayer are melamine formaldehyde spheres (Microparticles $\mathrm{GmbH}$ ) of diameter $\sigma_{s}=2.7 \mu \mathrm{m}$, dispersed in a 20/80 $v / v \%$ isopropanol/water mixture. The added impurities are melamine formaldehyde spheres of diameter $\sigma_{i}=6.1 \mu \mathrm{m}$ or $10.8 \mu \mathrm{m}$. The particles are contained in a $200 \mu \mathrm{m}$ thick quartz cell (Hellma Analytics) and due to their high mass density $\left(1.51 \mathrm{~g} / \mathrm{cm}^{3}\right)$, they sediment to the bottom wall of the cell within a few minutes to form a monolayer. In these conditions, it has been showed that these particles behave as hard spheres in a quasi twodimensional system as the out-of-plane fluctuations are negligible. ${ }^{31}$

Impurity-doped polycrystalline monolayers are prepared with different concentrations of impurities. The sample cell is placed on a levelled microscope stage (Olympus CKX41) and the polycrystalline monolayer is left to coarsen until grain growth has stopped due to the presence of impurities. All the samples are prepared 24 hours before data acquisition to ensure this stationary regime has been reached. Images of the polycrystalline structures are recorded using a $20 \times$ objective and a CMOS camera (Ximea XiQ). As the area of the sample cell is much bigger than the area of interest, images containing $\sim 4 \times 10^{4}$ particles are taken at different locations within the monolayer to improve statistics for a given concentration of impurities. This is necessary as the fluctuations in the number of impurities per frame can be significant, especially when their concentration is small.

\section{Composition variables}

The confinement of the host and impurity particles to a monolayer leads to non-additivity as the effective impurity size in the plane of the smaller host particles, $\sigma_{i}^{\prime}$, can be significantly 
(a)

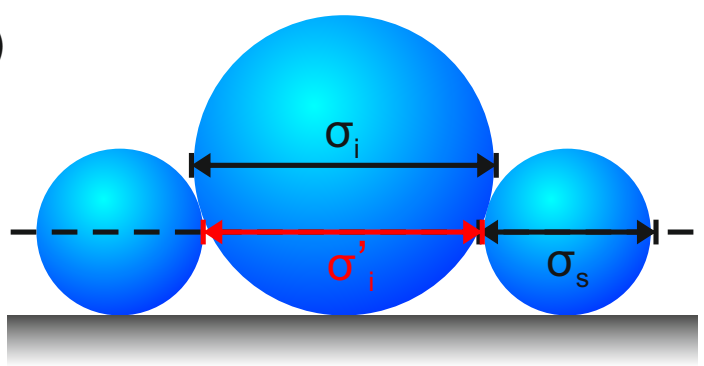

(b)

\begin{tabular}{c|c|c|c}
$\sigma_{\mathrm{i}}(\mu \mathrm{m})$ & $\lambda$ & $\lambda^{\prime}$ & $\Delta$ \\
\hline \hline 6.1 & 2.26 & 2 & -0.08 \\
\hline 10.8 & 4 & 3 & -0.2
\end{tabular}

Figure 1: Schematic view of close-packed hard spheres of two different sizes arranged in a monolayer. The diameters of the small and large particles are denoted $\sigma_{s}$ and $\sigma_{i}$ respectively. The effective diameter in the plane of the small particles (dashed line) is denoted $\sigma_{i}^{\prime}$. (b) Characteristics of the two types of impurity particles: diameter $\sigma_{i}$, real and in-plane size ratios $\lambda$ and $\lambda^{\prime}$, together with the non-additivity parameter $\Delta$ (Eq. (4)).

different from the actual impurity size, $\sigma_{i}$ (see Fig. 1(a)). Using elementary geometry, the effective in-plane impurity size, $\sigma_{i}^{\prime}$ in Fig. $1(\mathrm{a})$, is found as

$$
\sigma_{i}^{\prime}=\sigma_{s}\left(2 \sqrt{\frac{\sigma_{i}}{\sigma_{s}}}-1\right) .
$$

The non-additivity is quantified by the nonadditivity parameter, $\Delta$, defined by ${ }^{29,30}$

$$
\frac{\sigma_{s}+\sigma_{i}^{\prime}}{2}=\frac{\sigma_{s}+\sigma_{i}}{2}(1+\Delta) .
$$

Defining the actual and in-plane size ratios between the impurities and the host particles as $\lambda=\sigma_{i} / \sigma_{s}$ and $\lambda^{\prime}=\sigma_{i}^{\prime} / \sigma_{s}$, the non-additivity parameter can be written as

$$
\Delta=\frac{\lambda^{\prime}-\lambda}{\lambda+1} .
$$

The characteristic geometrical quantities of the two types of impurities are given in Fig. 1(b), showing that $\Delta$ is negative and that the inplane size ratios are integers in both cases.

To characterise the partitioning of impurities between the bulk and the grain boundaries, we define the composition of the bulk crystal, $Q_{b}$, and that of the GBs, $Q_{g b}$, by

$$
\begin{aligned}
Q_{b} & =\frac{\lambda^{\prime 2} N_{i}^{b}}{N_{s}^{b}+\lambda^{\prime 2} N_{i}^{b}}, \\
Q_{g b} & =\frac{\lambda^{\prime 2} N_{i}^{g b}}{N_{s}^{g b}+\lambda^{\prime 2} N_{i}^{g b}} .
\end{aligned}
$$

Here, $N_{s}^{b}\left(N_{s}^{g b}\right)$ is the number of host particles in the bulk (GBs,) and $N_{i}^{b}\left(N_{i}^{g b}\right)$ the number of impurities in the bulk (GBs). Note that both $Q_{b}$ and $Q_{g b}$ vary between 0 and 1, corresponding to the cases where no or only impurities are present in the bulk crystal and the GBs, respectively.

\section{Detection of adsorbed impurities}

Standard image analysis routines are used to obtain the coordinates of the particles in each frame ${ }^{32}$ and the impurity particles are easily discriminated from the host particles based on their size and brightness, see Fig. 2(a).

To quantify the local crystalline order of a particle $j$, we use the bond-orientational order parameter defined by ${ }^{33}$

$$
\psi_{6}\left(\vec{r}_{j}\right)=\frac{1}{N_{j}} \sum_{k=1}^{N_{j}} e^{i 6 \Delta \theta_{j k}},
$$

where $\Delta \theta_{j k}$ is the angle between the $x$-direction and the bond vector connecting the particle $j$ to one of its $N_{j}$ nearest neighbours $k$, defined using a Delaunay triangulation (Fig. 2(a), inset). The $\psi_{6}$-field is subsequently coarse-grained by replacing its value at $\vec{r}_{j}$ by the average over the two shells of nearest neighbours of the particle $j$ (Fig. 2(a), inset), which helps defining the grains better by removing small fluctuations of $\psi_{6}$. The local orientation is then given by $\theta_{6}=\arg \left(\psi_{6}\right) / 6$, and varies from 0 to $60^{\circ}$ due to the hexagonal symmetry of the $2 \mathrm{D}$ crystal. An example of the local orientation map is shown in Fig. 2(b) for $\lambda^{\prime}=3$, where an impurity in a 

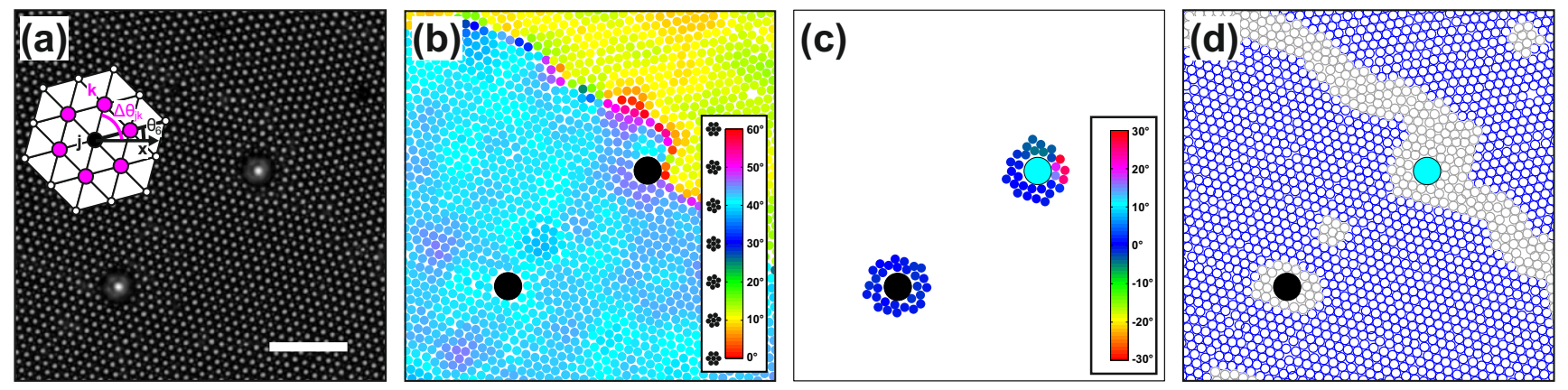

Figure 2: (a) Image of a small area of a colloidal polycrystalline monolayer containing impurities of size ratio $\lambda^{\prime}=3$. The inset shows the parameters used to define $\psi_{6}$ and $\theta_{6}$ (see text). Scale bar, $30 \mu \mathrm{m}$. (b) Output of the particle detection where impurities are black disks and host particles are coloured according to their orientation $\theta_{6}$, indicated by the colourbar. (c) The impurities with their two shells of nearest neighbours, coloured according to the value of their orientation relative to the mean, $\theta_{6}^{0}$ (see Eq. (7)), as indicated by the colourbar. The impurity successfully detected as belonging to the grain boundary is coloured in cyan. (d) The crystal and GB host particles coloured in blue and grey, respectively, with the two discriminated impurities.

grain and one in a GB can be seen.

To determine whether impurities are located in grains or adsorbed at GBs, a procedure is developed based on the local orientation around impurities, as this is uniform if they lie inside a grain and strongly varies if they are located at a GB. Firstly, for every impurity, the two shells of nearest neighbours are selected, a set denoted $S_{2}$ (see Fig. 2(c)). Next, for each particle $j \in S_{2}$, the orientation relative to the mean is defined as

$$
\theta_{6}^{0}\left(\vec{r}_{j}\right)=\frac{1}{6} \arg \left(\frac{\psi_{6}\left(\vec{r}_{j}\right)}{\left\langle\psi_{6}\left(\vec{r}_{k}\right)\right\rangle_{k \in S_{2}}}\right),
$$

where $\langle$.$\rangle denotes the average over S_{2}$ and the argument is taken in the interval $(-\pi / 6 ; \pi / 6]$. By definition, $\theta_{6}^{0}$ is always distributed around 0 , which avoids having the discontinuity between 0 and $\pi / 3$ present in the $\theta_{6}$-field due to the 6 -fold symmetry. The neighbours of impurity particles adsorbed at GBs typically have large values of $\theta_{6}^{0}$, while neighbours of bulk impurities have $\theta_{6}^{0}$ values close to 0 , as can be seen from Fig. 2(c). Finally, an impurity is considered as adsorbed at a GB if at least 3 particles in its neighbourhood $S_{2}$ have $\left|\theta_{6}^{0}\right|>3^{\circ}$. The value of this cut-off was chosen by trial and error on representative snapshots, and Fig. 2(d) shows that the impurity adsorbed in the GB is successfully identified by the algorithm. When performed on a whole snapshot, this analysis enables the direct measurement of the number of impurities in the bulk, $N_{i}^{b}$, and in the GBs, $N_{i}^{g b}$.

To discriminate between the host particles belonging to crystalline grains and the GBs, the following criterion is applied: a host particle $j$ is considered to be in a GB if $\left|\psi_{6}\left(\vec{r}_{j}\right)\right|<0.7$, otherwise, it belongs to a crystalline grain. ${ }^{34}$ Figure 2(d) shows the crystalline host particles in blue and the GB ones in grey. The latter correspond well to the GBs, but also include distorted layers around bulk impurities. The numbers of host particles in the bulk crystal and in the GBs, $N_{s}^{b}$ and $N_{s}^{g b}$, together with $N_{i}^{b}$ and $N_{i}^{g b}$, are used to determine the composition of the bulk and the GBs, $Q_{b}$ and $Q_{g b}$, according to Eq. (5a) and Eq. (5b).

\section{Results and discussion}

\section{Characterisation of impurities}

We first characterise the behaviour of the impurities in the pure crystal by considering microscopy images of isolated impurities embedded in the bulk crystal for both size ratios, see Fig. 3(a). The corresponding computer reconstructions of these two snapshots are shown in Fig. 3(b), where each particle is represented by a disk with the relevant hard core diameter. For 


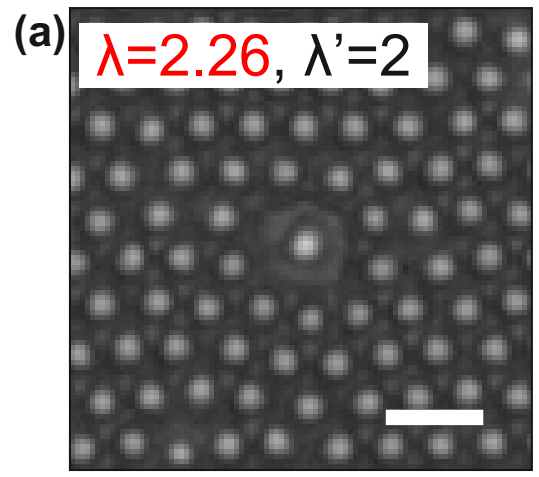

(b)

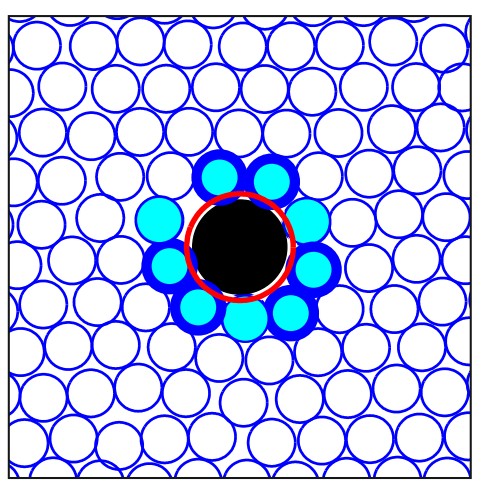

(c)
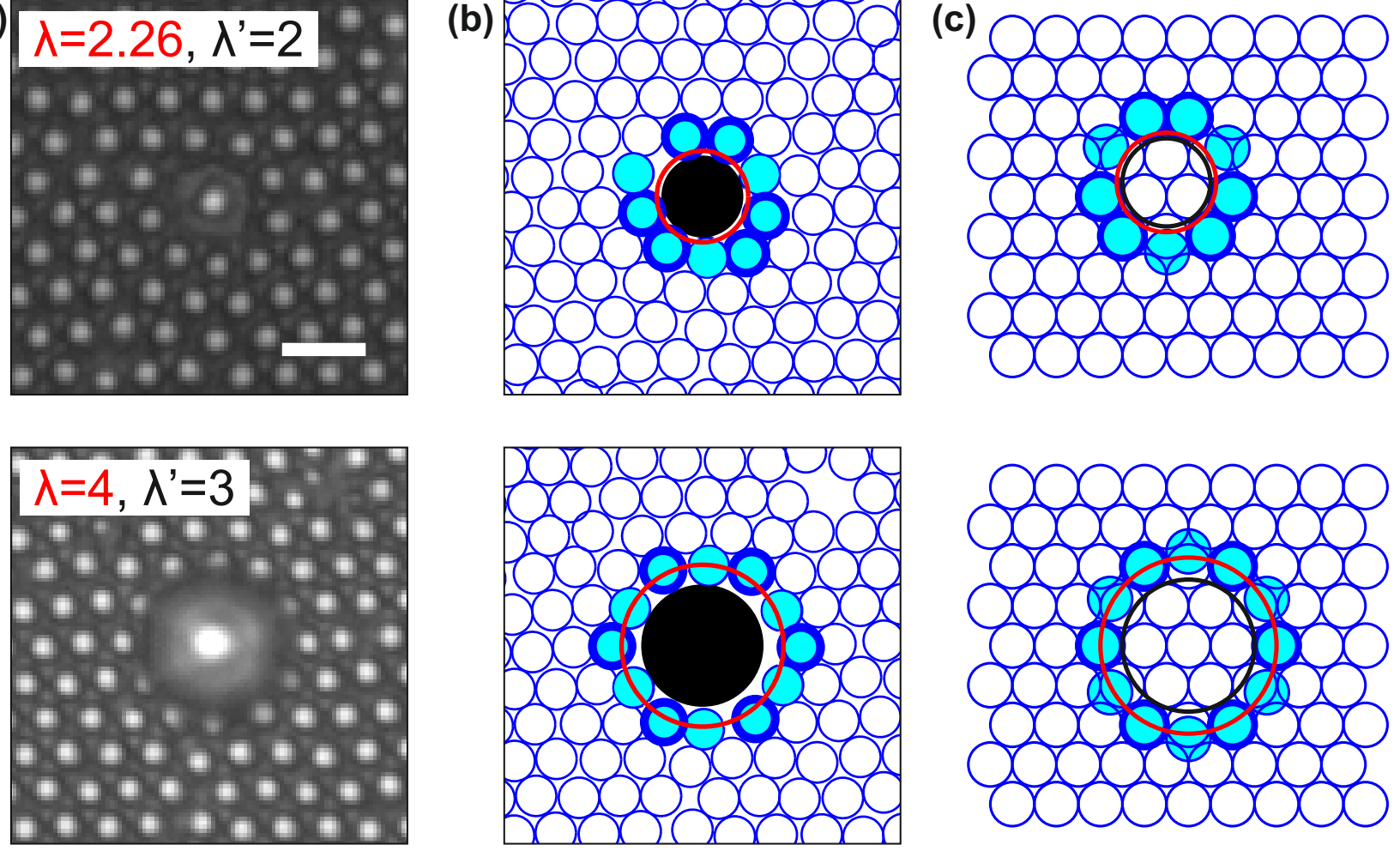

Figure 3: (a) Microscopy images of isolated impurities of each size embedded in the crystal. Scale bar, $5 \mu \mathrm{m}$. (b) Corresponding reconstructions of the images where each particle is represented by a disk with the relevant hard sphere diameter. Host particles are in blue with diameter $\sigma_{s}$, and the impurities are represented by a black disk of diameter $\sigma_{i}^{\prime}$ and a red circle of diameter $\sigma_{i}$. The first shell of nearest neighbours is coloured in cyan, and the thick circles highlight particles in coincidence with the crystal. (c) Equivalent geometric constructions on an ideal lattice with the same colour code, showing that the center of mass of an impurity with $\lambda^{\prime}=2$ occupies an interstitial site, while it occupies a crystal site for an impurity with $\lambda^{\prime}=3$.

the host particles (blue) this is $\sigma_{s}$, while for the impurity particles two disks of diameters $\sigma_{i}$ (the actual size, in red) and $\sigma_{i}^{\prime}$ (the in-plane size defined in Eq. (2), in black) are used. The nearest neighbours (cyan) are arranged in a ring around the impurities, which is partly distorted compared to the bulk crystal structure, though the latter is recovered at longer distances. ${ }^{19,35}$ Note that the overhang results in the host particles next to the impurities $\left(\lambda^{\prime}=3\right)$ appearing slightly dimmer and smaller. We verified using inverted confocal microscopy in reflection mode ${ }^{36}$ that these particles are located in the same plane as the other host particles (see Supporting Information, Fig. S1).

Interestingly, none of the impurities introduce any dislocations in the lattice as Burgers circuits drawn around them close up in a perfect lattice. ${ }^{33}$ This is consistent with these impurities either being located at a lattice site or an interstitial site. We discriminated between these two cases by overlaying a disk of diameter $\sigma_{i}^{\prime}$ on a perfect hexagonal lattice and looking for the configuration that maximises the number of nearest neighbours that coincide with bulk lattice sites (thick circles in Fig. 3(b) and (c)). The best matching situations are shown in Fig. 3(c), where the impurity is located at an interstitial site in the case of $\lambda^{\prime}=2$, and at a lattice site in the case of $\lambda^{\prime}=3$. In fact, these plots show that impurities with $\lambda^{\prime}=2$ behave as interstitial impurities, while impurities with $\lambda^{\prime}=3$ behave as substitutional impurities. We use this terminology, which is based on their behaviour within the crystalline grains, to distinguish the impurities throughout the remainder of the pa- 


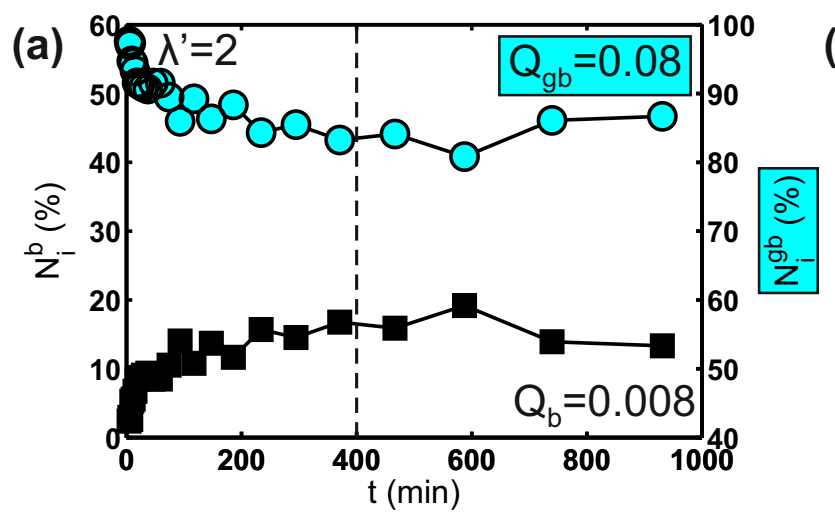

(b)
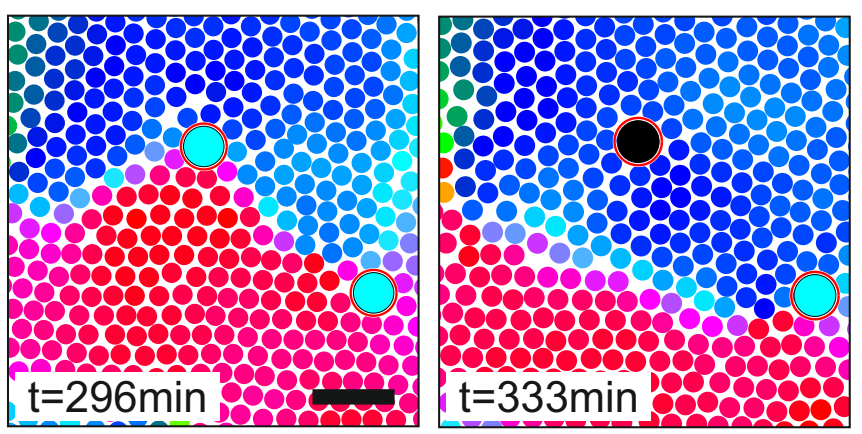

Figure 4: (a) Time evolution of the number of impurities within the grains, $N_{i}^{b}$, and that of impurities adsorbed at GBs, $N_{i}^{g b}$, expressed as a percentage of the total number of impurities, $N_{i}^{b}+N_{i}^{g b}$. From $t \simeq 400$ min after sample preparation (dashed line), a stationary regime is reached (see Movie S1). The compositions of the grains and the GBs (see Eq. (5a) and Eq. (5b)) are given 24 hours after sample preparation, when the stationary regime is well established. (b) De-pinning of an impurity during GB migration (see Movie S2). The impurities are shown in black if in a grain and in cyan if adsorbed at a GB. Scale bar, $10 \mu \mathrm{m}$.

per. Note that in metallurgy, however, interstitial impurities are usually smaller than the atoms constituting the crystal, in contrast to the present case.

\section{Dynamics of segregation during grain growth}

In order to confirm that a stationary regime has been reached 24 hours after sample preparation, we monitor the dynamics of impurity partitioning during grain growth. Figure 4(a) shows the time evolution of $N_{i}^{b}$ and $N_{i}^{g b}$ until about 16 hours after sample preparation, in the case of $\lambda^{\prime}=2$. One can clearly see that both curves flatten after $t \simeq 400 \mathrm{~min}$, indicating that a stationary regime has been reached with constant impurity compositions in the bulk grains and GBs (see Movie S1). Thus by measuring the compositions of the grains and the GBs, $Q_{b}$ and $Q_{g b}, 24$ hours after sample preparation, we ensure that these values corresponds to those in the stationary regime.

Figure 4(a) also gives important insight into the dynamics of the segregation process. Indeed, it clearly shows that $N_{i}^{b}$ increases in time while $N_{i}^{g b}$ decreases, indicating that, effectively, a fraction of impurities initially in GBs ends up in the bulk grains. This happens by de-pinning of impurities initially located in GBs during GB migration as shown in Fig. 4(b) and Movie S2. The stationary regime is reached when the average curvature of GBs is so low that GB migration cannot overcome the pinning force, ${ }^{1}$ which causes grain growth to stop (see Movie S1).

\section{Equilibrium segregation and ad- sorption isotherms}

Figure 5 shows partial views of polycrystalline structures in the stationary regime for three values of the bulk composition, $Q_{b}=10^{-3}, 10^{-2}$ and $10^{-1}$. The impurities are coloured in black if embedded in a grain and in cyan if adsorbed at a GB. Most impurities clearly populate the GBs, which indicates that GB segregation is occurring in the system. The tendency of impurities to segregate at GBs can be explained by the fact that the strain energy is much lower in the case of an impurity located at a GB than in the case of an impurity embedded in the bulk crystal. ${ }^{12}$ The grain size is observed to decrease with increasing $Q_{b}$, confirming that the addition of impurities is a way to control the grain size. ${ }^{23,37}$ Notably, at $Q_{b}=10^{-3}$ and for both size ratios, only a few impurity particles are adsorbed at GBs, which is already sufficient to stop grain growth. Next, for $Q_{b}=10^{-2}$, signif- 

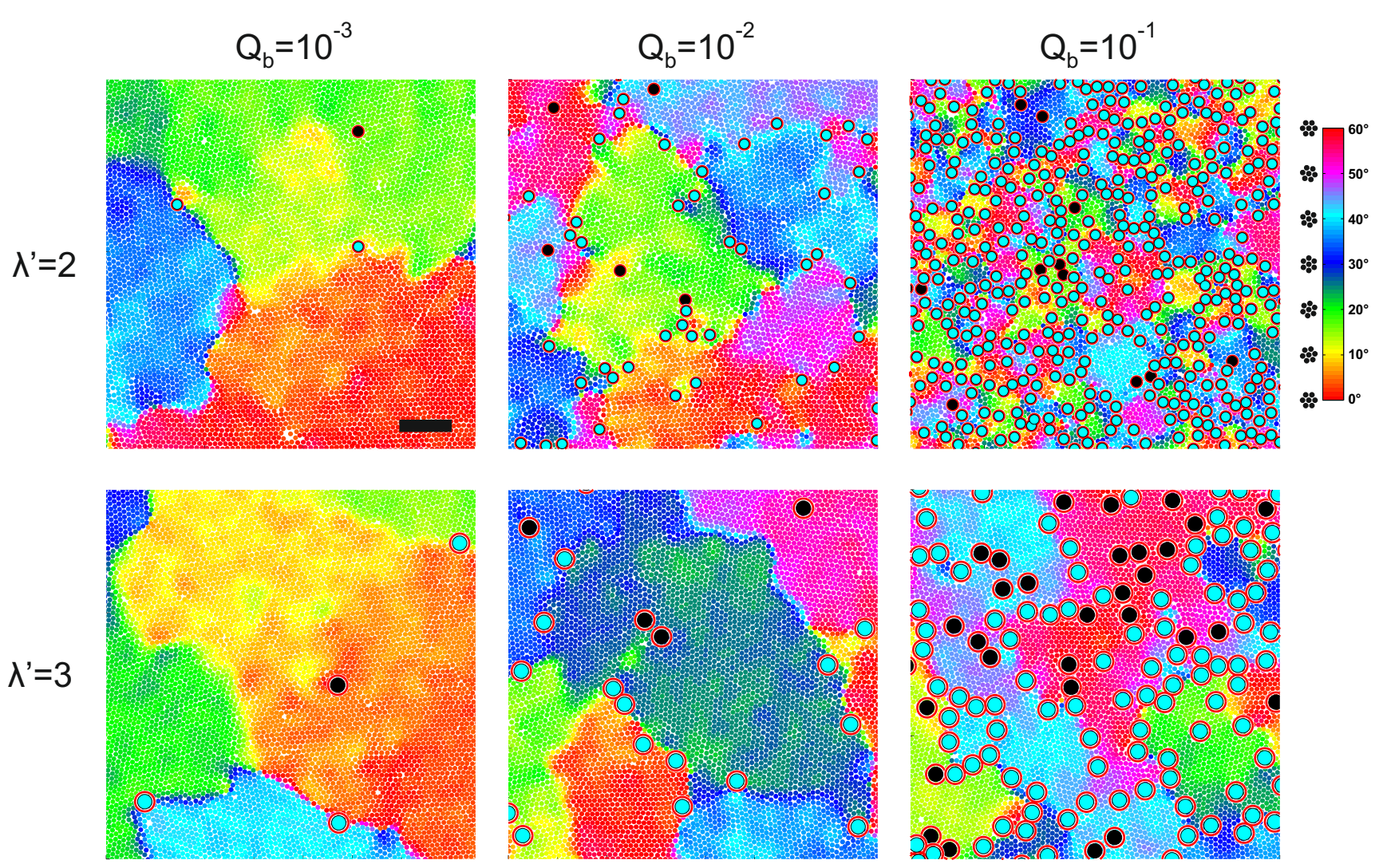

Figure 5: Partial views of the polycrystalline monolayer for the two types of impurities, characterised by their in-plane size ratio $\lambda^{\prime}$, at three different bulk impurity compositions $Q_{b}$. The host particles are coloured according to their local orientation (see colourbar) while the impurities are shown in black if in a grain and in cyan if adsorbed at a GB. The red circles correspond to the actual dimensions of the impurities. Scale bar, $30 \mu \mathrm{m}$.

icantly more impurities are located at the GBs compared to the number of impurities in the grains, clearly indicating further GB segregation, while the sample remains polycrystalline. Finally, in the case of $Q_{b}=10^{-1}$, the GBs are loaded with many impurities and the polycrystalline nature is preserved. Note, however, that some areas of the sample look quite disordered, especially for $Q_{b}=10^{-1}$ and $\lambda^{\prime}=2$. This is consistent with a recent study on colloidal microgels, which showed that high impurity concentrations can lead to vitrification. ${ }^{38}$

Next, we quantify the GB segregation by determining the adsorption isotherms, i.e. plotting $Q_{g b}$ as a function of $Q_{b}$, as shown in Fig. 6(a) and (b) for interstitial $\left(\lambda^{\prime}=2\right)$ and substitutional $\left(\lambda^{\prime}=3\right)$ impurities, respectively. In both cases, the data covers the dilute regime, where $Q_{g b}$ increases linearly up to $Q_{b} \sim 10^{-2}$. A more concentrated regime can be observed, where the curves flatten approaching saturation around $Q_{b} \sim 10^{-1}$. The solid lines correspond to fits to the LangmuirMcLean isotherm, Eq. (1), for each size ratio, but now rewritten in terms of composition variables:

$$
Q_{g b}=Q_{g b}^{s a t} \frac{K Q_{b}}{1+K Q_{b}} .
$$

Here, the GB composition at saturation, $Q_{g b}^{s a t}$, and the equilibrium constant, $K$, are the fitting parameters. As can be seen from Fig. 6(a) and (b), the Langmuir-McLean model describes the experimental data very well, indicating that equilibrium between the impurity content of the GBs and the grains has been reached.

The saturation levels for the two size ratios are very similar as the fits give $Q_{g b}^{s a t}=0.40 \pm 0.07$ for $\lambda^{\prime}=2$ and $0.37 \pm 0.07$ 
(a)

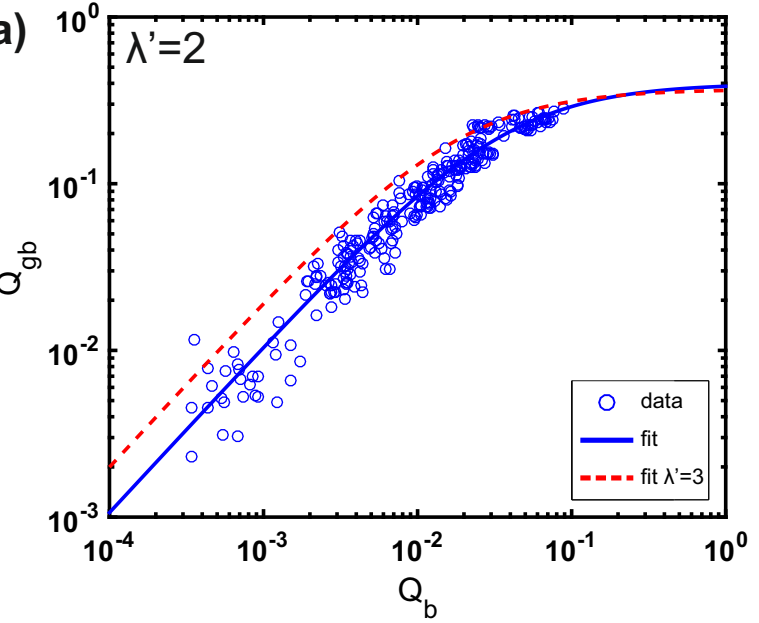

(b)

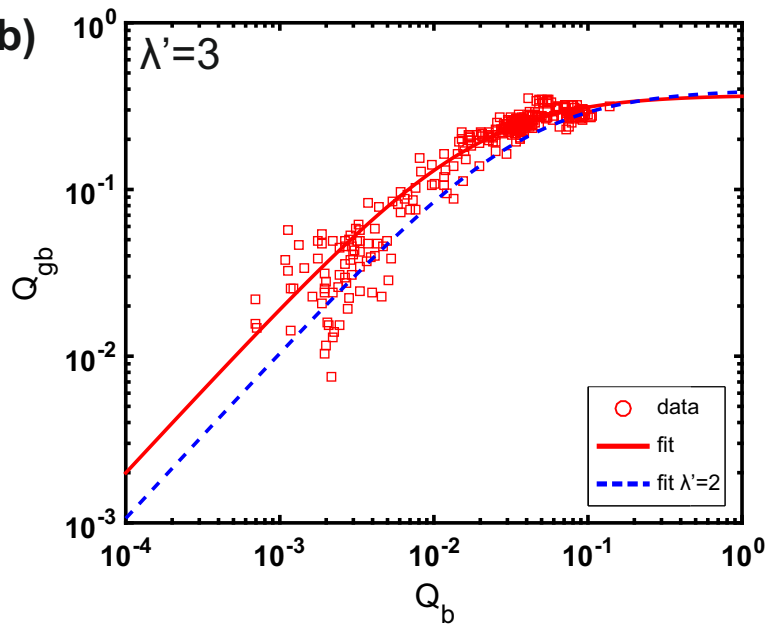

Figure 6: (a) Variation of the GB composition $Q_{g b}$ as a function of the bulk composition $Q_{b}$ for interstitial impurities $\left(\lambda^{\prime}=2\right)$. (b) Same data for substitutional impurities $\left(\lambda^{\prime}=3\right)$. The solid lines are fits to the Langmuir-McLean theory and the dashed lines correspond to the fitted curve for the other size ratio on each graph to allow for direct comparison. The fits give equilibrium constants of $K=26.76 \pm 3.17$ and $54.33 \pm 5.93$ for $\lambda^{\prime}=2$ and 3, respectively.

for $\lambda^{\prime}=3$. Interestingly, these values are significantly below unity, indicating that saturation does not correspond to a GB fully covered by impurities. In fact, the majority of the distorted sites constituting the GBs are still vacant as $Q_{g b}^{s a t}$ is well below 0.5. Studies of GB segregation in metals also report partial coverage at saturation in most cases. ${ }^{12-14}$

Importantly, we can directly determine the free energy of adsorption from the isotherm via $e_{a} / k_{B} T=\ln (K)$. The obtained adsorption free energies are $e_{a} / k_{B} T=3.29 \pm 0.12$ for $\lambda^{\prime}=2$ and $4.00 \pm 0.11$ for $\lambda^{\prime}=3$. Thus the large impurities have a larger adsorption free energy than the small impurities, leading to a higher coverage of the GBs at equal $Q_{b}$ (see Fig. 6). Interestingly, the adsorption free energy has an entropic origin as there are only hard core interactions in this system. The value of the adsorption free energy is only a few $k_{B} T$ in both cases, indicating that adsorption at GBs is reversible. This corroborates the idea that the observed GB segregation corresponds to equilibrium segregation, as already suggested by the agreement with the Langmuir-McLean model.

Overall, the interstitial or substitutional character of the impurities has little impact on the values of $Q_{g b}^{s a t}$ and $e_{a}$, which are in fact fairly comparable for both cases. Indeed, in our hard sphere system, the main contribution to the strain energy is the size mismatch between the impurities and the host particles, $\lambda^{\prime}$. As the values of $\lambda^{\prime}$ for both types of impurities are fairly similar, the strain energies - and hence the values of $Q_{g b}^{s a t}$ and $e_{a}$ - are not expected to be very different. However, we show in the remainder of the paper that the interstitial or substitutional character induces structural differences concerning the impurities adsorbed at GBs.

\section{Distribution of impurities at grain boundaries}

\section{Pair correlation function}

Next, we analyse the spatial distribution of impurities in the GBs close to saturation for both size ratios, i.e. for interstitial $\left(\lambda^{\prime}=2\right)$ and substitutional impurities $\left(\lambda^{\prime}=3\right)$. To this end, the pair correlation function, $g(r)$, of the impurities located at GBs only is computed according to

$$
g(r)=\frac{\pi \sigma_{i}^{\prime 2}}{4 N_{i}^{g b} \phi_{i}^{g b}}\left\langle\sum_{j=1}^{N_{i}^{g b}} \sum_{k \neq j} \delta\left(\vec{r}-\vec{r}_{j}+\vec{r}_{k}\right)\right\rangle,
$$

for samples having $Q_{g b}$ close to the saturation level. Here, $\phi_{i}^{g b}$ denotes the area fraction of adsorbed impurities defined by 
(a)

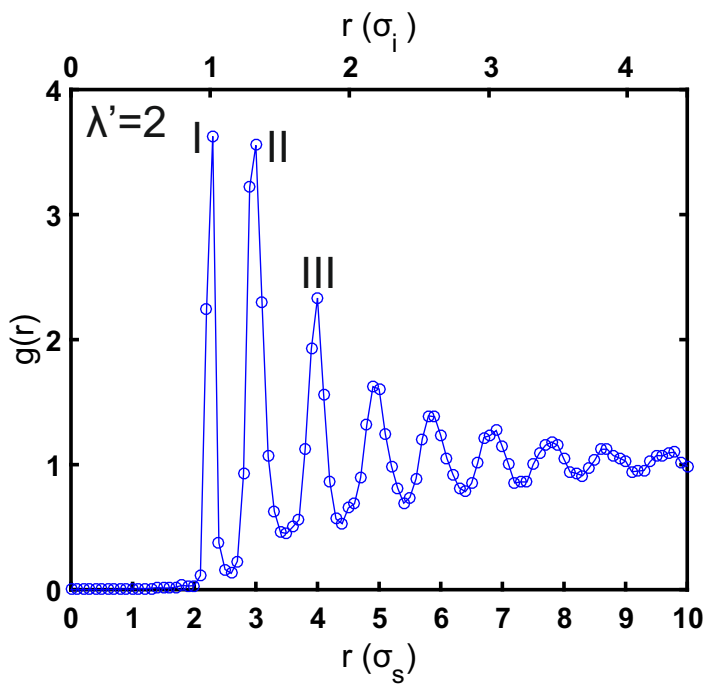

(c)

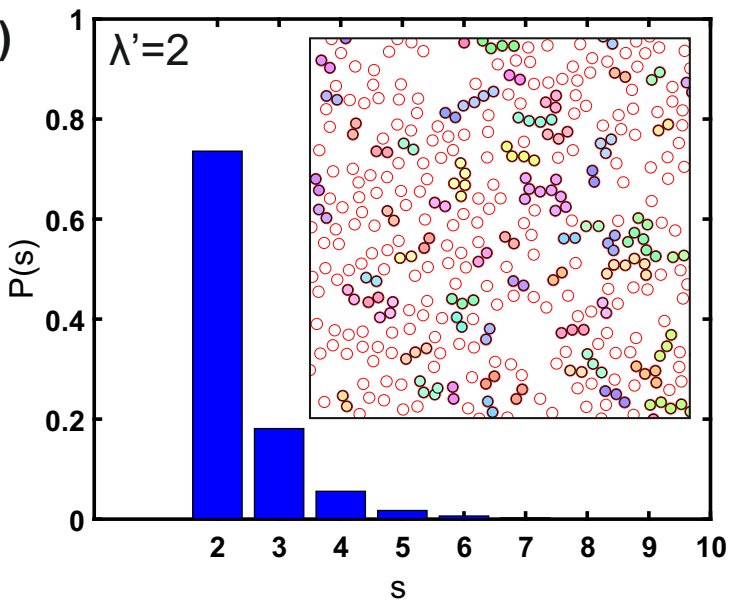

(b)

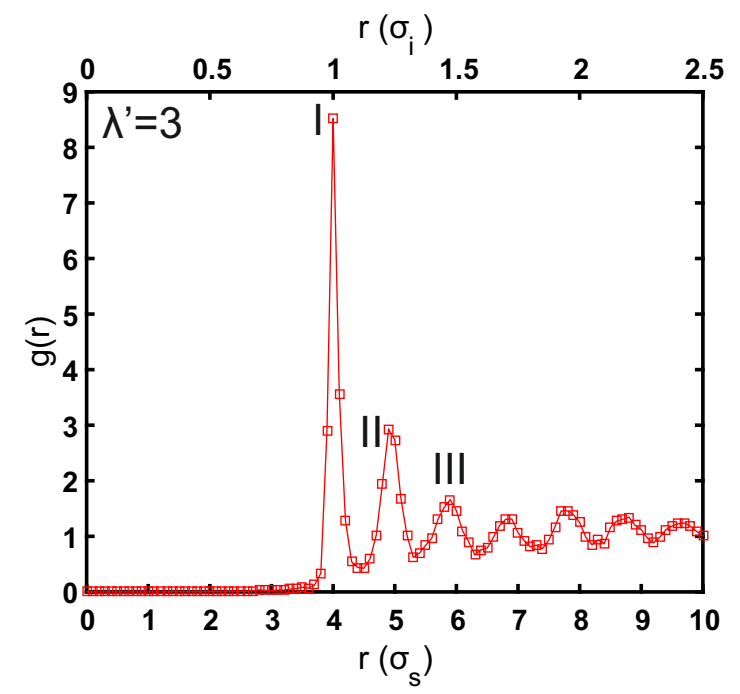

(d)

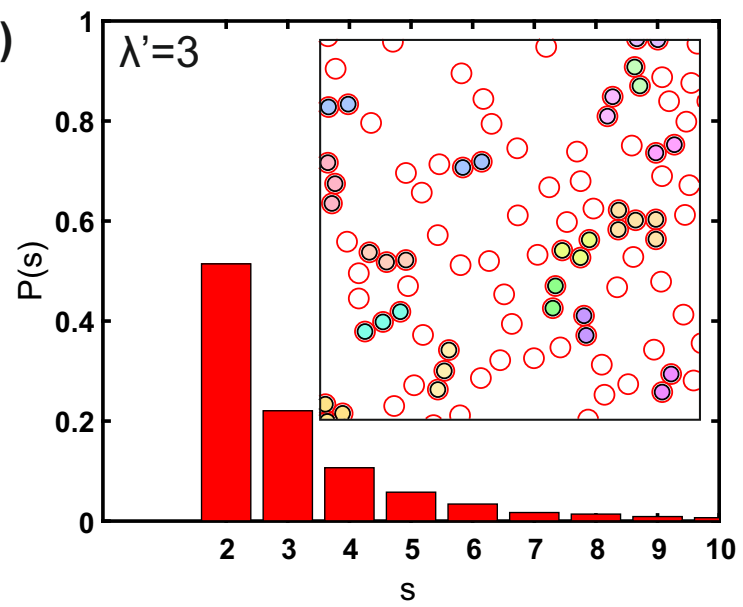

Figure 7: (a) Average pair correlation function of interstitial impurities $\left(\lambda^{\prime}=2\right)$ adsorbed at GBs for samples of composition $Q_{g b}=0.24 \pm 0.02$. The first three peaks (I, II and III) are described in the text. (b) The same quantity for the substitutional impurities $\left(\lambda^{\prime}=3\right)$ with $Q_{g b}=0.30 \pm 0.03$. (c) Size distribution of the clusters of two or more GB interstitial impurities $\left(\lambda^{\prime}=2\right)$, dominated by the presence of dimers. The data is the same as in (a). The inset shows a representative partial view of the clusters (colours) together with isolated impurities (circles), which corresponds to Fig. 5 for $Q_{b}=10^{-1}$ and $\lambda^{\prime}=2$. (d) Same as in (c) for substitutional impurities $\left(\lambda^{\prime}=3\right)$. The decay of the distribution is much smoother indicating the presence of larger clusters at the expense of dimers.

$\phi_{i}^{g b}=N_{i}^{g b} \pi \sigma_{i}^{\prime 2} /(4 A)$, where $A$ is the area of the field of view. Figure $7(\mathrm{a})$ shows the $g(r)$ of interstitial impurities $\left(\lambda^{\prime}=2\right)$ for $Q_{g b}=0.24 \pm 0.02$, and the same quantity is shown in Fig. 7(b) for substitutional impurities $\left(\lambda^{\prime}=3\right)$ at $Q_{g b}=0.30 \pm 0.03$. Note that, in all of the following, the distance $r$ is expressed in units of $\sigma_{s}$, which corresponds to the lattice spacing at close packing.

Importantly, a first examination of the relative magnitude of the peaks in the $g(r)$ for both size ratios in Fig. 7(a) and (b) shows that the distribution of impurities is structurally very different from a dilute 2D fluid of hard spheres at the same area fraction. ${ }^{31}$ For instance, in the case of interstitial impurities $\left(\lambda^{\prime}=2\right.$, Fig. 7(a)), the first and second peak have about the same magnitude. Moreover, for substitutional impurities $\left(\lambda^{\prime}=3\right.$, Fig. $\left.7(\mathrm{~b})\right)$, the first peak is about three times higher than the second. This very likely originates from the depletion forces caused by the host particles, ${ }^{29,30,39}$ leading to 
clustering of impurities in the GBs, as expected for systems with negative non-additivity parameters. ${ }^{27,28}$ Note that clustering is facilitated by the fact that the impurities are mobile and thus free to rearrange in the grain boundaries.

We now identify the origin of the peaks in the $g(r)$ for the two size ratios. For the interstitial $\left(\lambda^{\prime}=2\right)$ and substitutional $\left(\lambda^{\prime}=3\right)$ impurities, the first peak of $g(r)$ (I) is located at $r_{\mathrm{I}} \simeq 2.3$ and 4 , respectively, which exactly matches the value of $\lambda=2.26$ and 4 , in both cases. This peak thus originates from the direct contact between impurities, as $\lambda$ is the centreto-centre distance in this case, and corresponds to clustering of the impurities. However, for both size ratios, the second and third peaks (II and III) are not located at integer multiples of $\lambda$. They are thus incompatible with direct contact between the impurities and correspond to mixing with the host particles.

The relative importance of clustering and mixing seems to strongly depend on the size ratio as suggested by the clear differences in the $g(r)$ for both types of impurities. Indeed, the comparable magnitudes of the first and the second peak in the case of interstitial impurities $\left(\lambda^{\prime}=2\right.$, Fig. $\left.7(\mathrm{a})\right)$ suggests the presence of both clustering and mixing. Conversely, the high magnitude of the first peak compared to that of the second for substitutional impurities $\left(\lambda^{\prime}=3\right.$, Fig. $\left.7(\mathrm{~b})\right)$ indicates a prevalence of clustering. This underlines the importance of geometry in determining the distribution of impurities in our hard sphere system.

\section{Cluster formation}

To gain more insight into the role of depletion and clustering in the system, we analyse the clusters formed by both types of impurities. Two impurities are considered to be in the same cluster if their centre-to-centre distance is less than $\left(r_{\mathrm{I}}+r_{\mathrm{II}}\right) / 2$. The size distributions of the clusters, $P(s)$, as a function of the size, $s \geq 2$, are presented in Fig. 7(c) and (d) for the two types of impurities, respectively. The insets show the output of the cluster analysis for the images in Fig. 5 at $Q_{b}=10^{-1}$. In the case of interstitial impurities $\left(\lambda^{\prime}=2\right)$, the for- mation of dimers $(s=2)$ is clearly preferred over the formation of clusters of size $s \geq 3$, as inferred from the sharp drop in the probability $P(s)$ above $s=2$ (Fig. $7(\mathrm{c})$ ). In contrast, the decay of $P(s)$ is much smoother for substitutional impurities $\left(\lambda^{\prime}=3\right)$, which indicates that larger clusters are more frequent in this case (Fig. $7(\mathrm{~d})$ ). In the following, we give simple geometric arguments to rationalise the prevalence of dimers in the case of interstitial impurities $\left(\lambda^{\prime}=2\right)$ relative to that of substitutional impurities $\left(\lambda^{\prime}=3\right)$.

Interstitial impurities $\left(\lambda^{\prime}=2\right)$ The first peak of $g(r)(\mathrm{I})$ corresponds to configuration I in Fig. 8(a): the two impurities form a dimer with two host particles on either side. One can see that such a configuration consists of four close-packed particles and for this reason, it is reasonable to expect that their positions can be mapped onto a perfect hexagonal closed-packed lattice. Indeed, it turns out that an equivalent configuration exists on a perfect lattice, with two impurities at interstitial sites and the two side particles in coincidence with crystal sites (see Fig. 8(b)). Note that the location of the two impurities are consistent with their interstitial nature. Using simple geometry, the distance in between the two impurities is found to be $d_{\mathrm{I}}=4 / \sqrt{3} \simeq 2.31$, which is in very good agreement with the experimental location of the first peak, $r_{\mathrm{I}} \simeq 2.3$.

The second peak in $g(r)$ (II) actually consists of a shoulder at $r_{\mathrm{II}}^{-}=2.9$ and a maximum at $r_{\mathrm{II}}^{+}=3$, which correspond to two configurations with no direct contact between impurities. The first configuration, denoted $\mathrm{II}^{-}$in Fig. 8(a), has a dimer of host particles in between the two impurities. Again, mapping this configuration onto a perfect lattice with the dimer in coincidence with two lattice sites and the two impurities located at interstitial sites leads to an ideal distance of $d_{\mathrm{II}}^{-}=10 / \sqrt{12} \simeq 2.9$, which is in perfect agreement with the observed $r_{\mathrm{II}}^{-}$. The second configuration, $\mathrm{II}^{+}$, corresponds to two impurities sitting at interstitial sites situated on the same row of lattice sites with a triplet of host particles on one side, separated by a distance $d_{\mathrm{II}}^{+}=3$ (see Fig. $8(\mathrm{a})$ and (b)). 
(a)

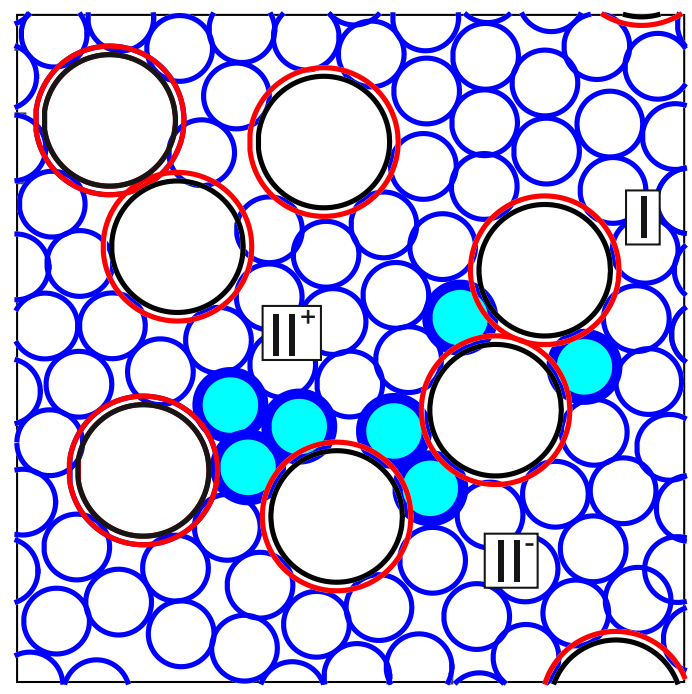

(c)

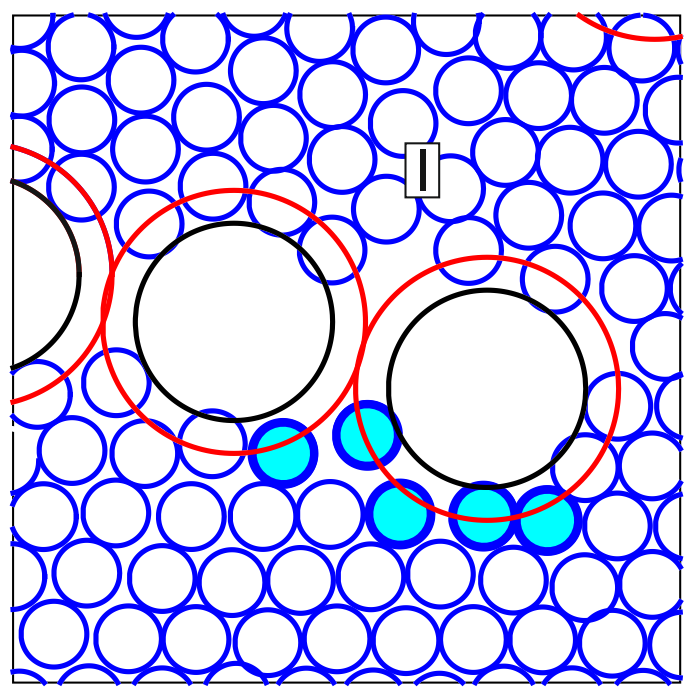

(b)

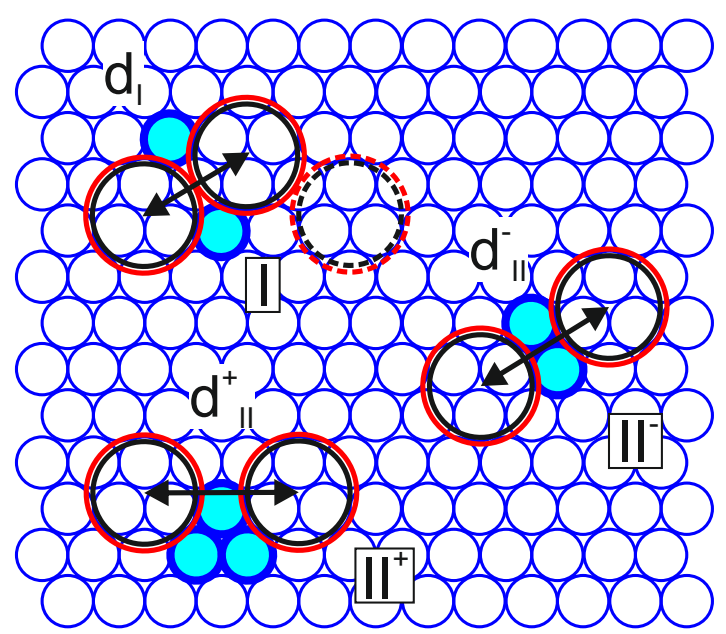

(d)

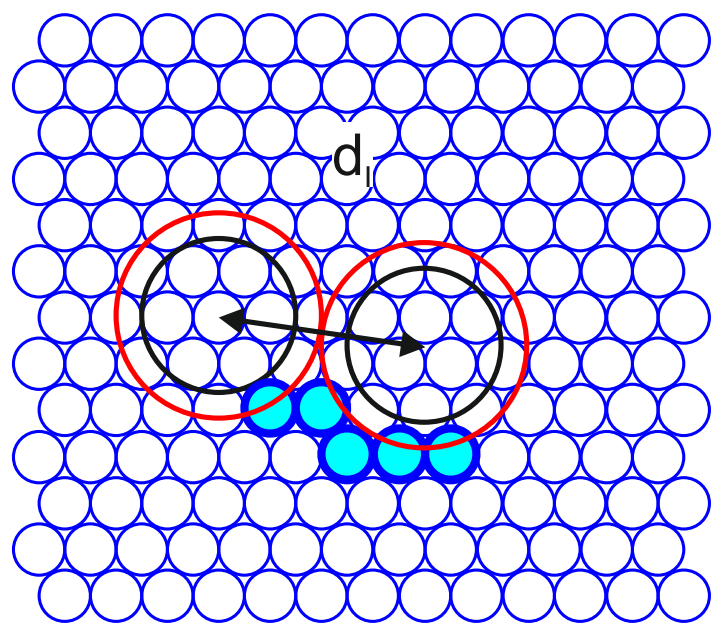

Figure 8: (a) Computer reconstruction of a local view of interstitial impurities $\left(\lambda^{\prime}=2\right)$ segregated at a GB. The three types of configurations contributing to the values of $g(r), \mathrm{I}^{-} \mathrm{II}^{-}$and $\mathrm{II}^{+}$are illustrated. (b) The same three configurations but now on a perfect lattice. The particles in coincidence with the lattice are highlighted in cyan and thick circles. This enables geometrically calculating the values of the distances in between impurities, $d_{\mathrm{I}}, d_{\mathrm{II}}^{-}$and $d_{\mathrm{II}}^{+}$. The dashed circles represent one of the few possible ways of adding an impurity to configuration I, due to geometrical restrictions imposed by the lattice. (c) and (d) Same representation for a possible configuration in the case of substitutional impurities $\left(\lambda^{\prime}=3\right)$.

Again, this agrees very well with the observed value for $r_{\text {II }}^{+}$.

Importantly, these three configurations (I, II ${ }^{-}$ and $\mathrm{II}^{+}$) compete against each other, as their associated values of $g(r)$ are comparable. Configuration I contributes to clustering, as it corresponds to direct contact between impurities. However, the lattice geometry strongly restricts the number of possibilities to add more impurities to the cluster (see Fig. 8(b)). This repre- sents the first source of inhibition for the formation of clusters larger than $s=2$. The second source is the fact that $\mathrm{II}^{-}$and $\mathrm{II}^{+}$break direct contact between the interstitial impurities, contributing to mixing and thus preventing further clustering. These two sources have a clear geometric origin and explain why dimers are relatively more frequent. Both sources of inhibition for the formation of clusters larger than $s=2$ can be seen as a form of geometric frus- 
tration due to the interplay between depletion, non-additivity and lattice geometry.

Substitutional impurities $\left(\lambda^{\prime}=3\right)$ The very high first peak in the $g(r)$ at $r_{\mathrm{I}} \simeq 4$ corresponds to direct contact as it is equal to $\lambda$. As the impurities are substitutional, the expected configuration is two impurities located at two crystal sites on the same crystal row separated by a distance $r=4$. Remarkably, many other configurations like the one presented in Fig. 8(c) are also found. In this case, the mapping onto a perfect lattice in Fig. 8(d) shows that one impurity sits on a crystal site and the other impurity sits at an interstitial site, right below the crystal row. The distance between the two impurities is then $d_{\mathrm{I}}=\sqrt{49 / 3} \simeq 4.04$, which is consistent with a contribution to the first peak, involved in clustering. Crucially, these configurations enable impurities to form larger clusters, in contrast with the interstitial impurities. It is also interesting to note that impurities can change from being substitutional to interstitial due to interactions with others impurities.

\section{Conclusion}

To summarise, colloidal alloys provide very interesting model systems to study grain boundary segregation using simple video-microscopy. We found that the size ratio between the impurities and the host particles determines the interstitial or substitutional nature of the impurities. In both cases, the adsorption isotherms agree very well with the Langmuir-McLean model of equilibrium grain boundary segregation and the measured free energy of adsorption is small enough to allow for reversible adsorption. We have obtained detailed insight into the configuration of impurities close to saturation of the GBs. The relative balance between clustering and mixing due to non-additivity is found to strongly depend on the type of impurity. This is due to geometrical constraints imposed by the lattice, which prevent the formation of clusters larger than two impurities for interstitial impurities, while for substitutional impurities, the lattice geometry allows the formation of larger clusters.

Acknowledgement The authors thank Alice Thorneywork and Roland Roth for useful discussions. The European Research Council (ERC) is acknowledged for financial support (ERC Starting Grant 279541-IMCOLMAT).

\section{References}

(1) Rollett, A.; Humphreys, F. J.; Rohrer, G. S.; Hatherly, M. Recrystallization and related annealing phenomena; Elsevier, 2004.

(2) Cahn, J. W. The impurity-drag effect in grain boundary motion. Acta Metall. Mater. 1962, 10, $789-798$.

(3) Gottstein, G.; Shvindlerman, L. S. Grain boundary migration in metals: thermodynamics, kinetics, applications; CRC press, 1999 .

(4) Smith, C. S. Grains, phases, and interphases: an interpretation of microstructure. Trans. Metall. Soc. AIME 1948, 175, 15-51.

(5) Nes, E.; Ryum, N.; Hunderi, O. On the Zener drag. Acta Metall. Mater. 1985, 33, $11-22$.

(6) Srolovitz, D. J.; Grest, G. S. Impurity effects on domain-growth kinetics. II. Potts model. Phys. Rev. B 1985, 32, 3021-3025.

(7) Hassold, G. N.; Holm, E. A.; Srolovitz, D. J. Effects of particle size on inhibited grain growth. Scripta Metall. Mater. 1990, 24, $101-106$.

(8) Yip, S. Nanocrystals: the strongest size. Nature 1998, 391, 532-533.

(9) Schiøtz, J.; Di Tolla, F. D.; Jacobsen, K. W. Softening of nanocrystalline metals at very small grain sizes. Nature 1998, 391, 561-563. 
(10) Lu, L.; Chen, X.; Huang, X.; Lu, K. Revealing the maximum strength in nanotwinned copper. Science 2009, 323, 607610.

(11) McLean, D. Grain boundaries in metals; Monographs on the physics and chemistry of materials; Clarendon Press, 1957.

(12) Sutton, A. P.; Balluffi, R. W. Interfaces in crystalline materials; Clarendon Press, 1995.

(13) Hondros, E. D.; Seah, M. P. The theory of grain boundary segregation in terms of surface adsorption analogues. Metall. Trans. A 1977, 8, 1363-1371.

(14) Seah, M. P. Grain boundary segregation. J. Phys. F: Met. Phys. 1980, 10, 1043.

(15) Langmuir, I. The adsorption of gases on plane surfaces of glass, mica and platinum. J. Am. Chem. Soc. 1918, 40, 1361-1403.

(16) Barnes, G.; Gentle, I. Interfacial science: an introduction; Oxford University Press, 2011.

(17) Erhart, H.; Grabke, H. J. Equilibrium segregation of phosphorus at grain boundaries of $\mathrm{Fe}-\mathrm{P}, \mathrm{Fe}-\mathrm{C}-\mathrm{P}, \mathrm{Fe}-\mathrm{Cr}-\mathrm{P}$, and $\mathrm{Fe}^{-}$ Cr-C-P alloys. Met. Sci. 1981, 15, 401408.

(18) Grabke, H. J. Surface and grain boundary segregation on and in iron and steels. ISIJ International 1989, 29, 529-538.

(19) de Villeneuve, V. W. A.; Derendorp, L.; Verboekend, D.; Vermolen, E. C. M.; Kegel, W. K.; Lekkerkerker, H. N. W.; Dullens, R. P. A. Grain boundary pinning in doped hard sphere crystals. Soft Matter 2009, 5, 2448-2452.

(20) Yoshizawa, K.; Okuzono, T.; Koga, T.; Taniji, T.; Yamanaka, J. Exclusion of impurity particles during grain growth in charged colloidal crystals. Langmuir 2011, 27, 13420-13427.
(21) Ghofraniha, N.; Tamborini, E.; Oberdisse, J.; Cipelletti, L.; Ramos, L. Grain refinement and partitioning of impurities in the grain boundaries of a colloidal polycrystal. Soft Matter 2012, 8, 6214-6219.

(22) Yoshizawa, K.; Toyotama, A.; Okuzono, T.; Yamanaka, J. Exclusion of impurity particles in charged colloidal crystals. Soft Matter 2014, 10, 3357-3361.

(23) Palberg, T.; Mönch, W.; Schwarz, J.; Leiderer, P. Grain size control in polycrystalline colloidal solids. J. Chem. Phys. 1995, 102.

(24) Nozawa, J.; Uda, S.; Naradate, Y.; Koizumi, H.; Fujiwara, K.; Toyotama, A.; Yamanaka, J. Impurity partitioning during colloidal crystallization. J. Phys. Chem. B 2013, 117, 5289-5295.

(25) Hu, S.; Nozawa, J.; Koizumi, H.; Fujiwara, K.; Uda, S. Grain boundary segregation of impurities during polycrystalline colloidal crystallization. Cryst. Growth Des. 2015, 15, 5685-5692.

(26) Nozawa, J.; Uda, S.; Hu, S.; Fujiwara, K.; Koizumi, H. Orientation-dependent impurity partitioning of colloidal crystals. $J$. Cryst. Growth 2016, 439, $13-18$.

(27) Hoffmann, N.; Ebert, F.; Likos, C. N.; Löwen, H.; Maret, G. Partial clustering in binary two-dimensional colloidal suspensions. Phys. Rev. Lett. 2006, 97, 078301.

(28) Ebert, F.; Maret, G.; Keim, P. Partial clustering prevents global crystallization in a binary 2D colloidal glass former. Eur. Phys. J. E 2009, 29, 311-318.

(29) Roth, R.; Evans, R. The depletion potential in non-additive hard-sphere mixtures. Europhys. Lett. 2001, 53, 271.

(30) Roth, R.; Evans, R.; Louis, A. A. Theory of asymmetric nonadditive binary hardsphere mixtures. Phys. Rev. E 2001, 64, 051202. 
(31) Thorneywork, A. L.; Roth, R.; Aarts, D. G. A. L.; Dullens, R. P. A. Radial distribution functions in a two-dimensional binary colloidal hard sphere system. J. Chem. Phys. 2014, 140, 161106.

(32) Blair, D.; Dufresne, E. The Matlab Particle Tracking Code Repository. Retrieved from http://physics.georgetown.edu/matlab/.

(33) Nelson, D. R. Defects and geometry in condensed matter physics; Cambridge University Press, 2002.

(34) Dillmann, P.; Maret, G.; Keim, P. Polycrystalline solidification in a quenched $2 \mathrm{D}$ colloidal system. J. Phys. Condens. Matter 2008, 20, 404216.

(35) de Villeneuve, V. W. A.; Dullens, R. P. A.; Aarts, D. G. A. L.; Groeneveld, E.; Scherff, J. H.; Kegel, W. K.; Lekkerkerker, H. N. W. Colloidal hard-sphere crystal growth frustrated by large spherical impurities. Science 2005, 309, 12311233 .

(36) Baumgartl, J.; Dullens, R. P. A.; Dijkstra, M.; Roth, R.; Bechinger, C. Experimental observation of structural crossover in binary mixtures of colloidal hard spheres. Phys. Rev. Lett. 2007, 98, 198303.

(37) Gray, A. T.; Mould, E.; Royall, C. P.; Williams, I. Structural characterisation of polycrystalline colloidal monolayers in the presence of aspherical impurities. J. Phys. Condens. Matter 2015, 27, 194108.

(38) Higler, R.; Appel, J.; Sprakel, J. Substitutional impurity-induced vitrification in microgel crystals. Soft Matter 2013, 9, $5372-5379$.

(39) Perera-Burgos, J. A.; MéndezAlcaraz, J. M.; Pérez-Ángel, G.; Castañeda Priego, R. Assessment of the micro-structure and depletion potentials in two-dimensional binary mixtures of additive hard-disks. J. Chem. Phys. 2016, 145 . 


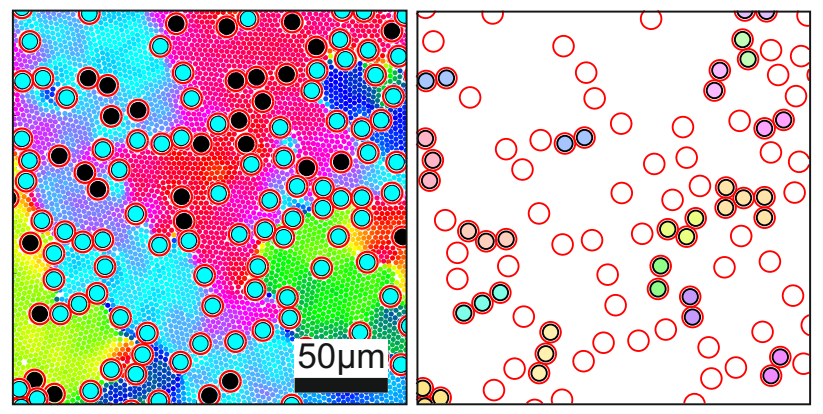

For Table of Contents Only. 